\title{
Analytical theory for the dark-soliton interaction in nonlocal nonlinear materials with an arbitrary degree of nonlocality
}

Kong, Qian; Wang, Q.; Bang, Ole; Krolikowski, W.

Published in:

Physical Review A

Link to article, DOI:

10.1103/PhysRevA.82.013826

Publication date:

2010

Document Version

Publisher's PDF, also known as Version of record

Link back to DTU Orbit

Citation (APA):

Kong, Q., Wang, Q., Bang, O., \& Krolikowski, W. (2010). Analytical theory for the dark-soliton interaction in nonlocal nonlinear materials with an arbitrary degree of nonlocality. Physical Review A, 82(1), 013826. https://doi.org/10.1103/PhysRevA.82.013826

\section{General rights}

Copyright and moral rights for the publications made accessible in the public portal are retained by the authors and/or other copyright owners and it is a condition of accessing publications that users recognise and abide by the legal requirements associated with these rights.

- Users may download and print one copy of any publication from the public portal for the purpose of private study or research.

- You may not further distribute the material or use it for any profit-making activity or commercial gain

- You may freely distribute the URL identifying the publication in the public portal 


\title{
Analytical theory for the dark-soliton interaction in nonlocal nonlinear materials with an arbitrary degree of nonlocality
}

\author{
Qian Kong, ${ }^{1,2}$ Q. Wang, ${ }^{2}$ O. Bang, ${ }^{3}$ and W. Krolikowski ${ }^{1}$ \\ ${ }^{1}$ Laser Physics Center, Research School of Physics and Engineering, Australian National University, \\ Canberra, Australian Capital Territory 0200, Australia \\ ${ }^{2}$ Department of Physics, Shanghai University, Shanghai 200444, China \\ ${ }^{3}$ DTU Fotonik, Department of Photonics Engineering, Technical University of Denmark, DK-2800 Kgs. Lyngby, Denmark
}

(Received 24 May 2010; published 21 July 2010)

\begin{abstract}
We investigate theoretically the interaction of dark solitons in materials with a spatially nonlocal nonlinearity. In particular we do this analytically and for arbitrary degree of nonlocality. We employ the variational technique to show that nonlocality induces an attractive force in the otherwise repulsive soliton interaction.
\end{abstract}

DOI: 10.1103/PhysRevA.82.013826

PACS number(s): 42.65.Tg, 42.65.Sf, 42.70.Df, 03.75.Lm

\section{INTRODUCTION}

Spatial optical solitons represent beams, which propagate in nonlinear media without changing their profile. Their existence is a result of an interplay between size-determined diffraction and nonlinearity-induced phase modulation, which in most cases is produced by the refractive index modification of the material. Depending on the type of nonlinearity, nonlinear media may support either bright or dark solitons [1]. While bright solitons are just finite-size beams formed in media with self-focusing nonlinearity, dark solitons are more complex objects, as they represent an intensity dip in an otherwise constant background with nontrivial phase profile [2]. Spatial dark solitons have been observed and studied in media with a negative or self-defocusing nonlinearity $[3,4]$. Their temporal counterparts, which have the form of "dark pulses" (i.e., temporal intensity dips on a cw background) can exist in optical fibers in the normal dispersion regime [5,6]. In recent years the renewed interest in the properties of dark solitons stem from experimental advances in the physics of matter waves. In particular, the formation of dark matter wave solitons has been observed in Bose-Einstein condensates with a repulsive interparticle interaction [7-10]. There has also been a report on the possibility of dark soliton formation in nonlinear metamaterials [10]. Interestingly, temporal dark solitons were also shown to be able to induce supercontinuum generation in photonic crystal fibers [11].

The unique property of optical solitons, either bright or dark, is their particle-like behavior in interaction [1]. However, it is also well known that there is a fundamental difference in the interaction of bright and dark solitons. While bright soltions may attract, repel, or even form bound states, depending on their relative phase [12-14], dark solitons always repel. This has been confirmed in numerous theoretical and experimental works [15-17]. We have shown recently that the nature of dark soliton interaction can be drastically altered by the spatially nonlocal character of nonlinearity $[18,19]$. In nonlocal media the nonlinear response of the medium in a particular spatial location is determined not only by the wave (light) intensity in that position, as in the local media, but also by the intensity in a certain neighborhood around the point. As a result, spatial nonlocality provides stabilization of bright solitons [20-22], and induces their attraction, even if they are out-of-phase [23,24]. Nonlocality has a similar effect on dark solitons. In particular, it has been shown both numerically [18] and experimentally [19] that nonlocality induces attraction of otherwise repelling dark solitons, leading to the formation of their bound states. The physics of soliton attraction in nonlocal nonlinear media can most easily be understood in the (linear) regime of strong nonlocality [20]. In the context of nonlinear optics, a strongly nonlocal response of the medium leads to the formation of a broad (linear) index waveguide, which can trap two or more solitons and enable the formation of bound states. In the context of matter waves such nonlocal (dipolar) interaction leads to the formation of a potential well, which again induces attraction between solitons. While dark soliton attraction has already been observed experimentally [19] the theoretical analysis of this phenomenon in the regime of arbitrary degree of nonlocality has been conducted only numerically [18] or analytically in the special linear regime of strong nonlocality [25].

In this work we will investigate analytically the interaction of dark solitons in nonlocal media with an arbitrary degree of nonlocality. We will consider a suitable nonlocal response function and use the variational approach to derive analytical formulas for the forces acting between two dark solitons. Our results clearly show how nonlocality induces an attractive force, which depends on the degree of nonlocality and counteracts the otherwise inherent repulsive nature of dark soliton interaction.

\section{THE NONLOCAL MODEL AND THE RESPONSE FUNCTION}

In what follows we will be interested in the evolution of $1+1$-dimensional optical beams with a scalar amplitude $E(x, z)$ and intensity $I(x, z)=|E(x, z)|^{2}$, that depends on the transverse $x$ coordinate and the propagation coordinate $z$. Propagation of such beams in materials with a nonlocal defocusing nonlinearity can be modeled by the following generic dimensionless nonlocal nonlinear Schrödinger (NLS) equation

$$
i \frac{\partial E}{\partial z}+\frac{1}{2} \frac{\partial^{2} E}{\partial x^{2}}-E \int_{-\infty}^{+\infty} R(x-\xi) I(\xi, z) d \xi=0,
$$

with the nonlocal response in the form of a convolution, where $R(x)$ is the nonlocal response function. In what follows we will use the normalization $\int_{-\infty}^{\infty} R(x) d x=1$. Obviously $R(x)=\delta(x)$ in a local Kerr medium. The actual form of the 
nonlocal response is determined by the details of the physical process responsible for the nonlocality. For all diffusion-type nonlinearities [26], orientational-type nonlinearities (like nematic liquid crystal) [23], and for the general quadratic nonlinearity describing parametric interaction [27-30], the response function is an exponential $R(x)=(2 \sigma)^{-1} \exp (-|x| / \sigma)$ originating from a Lorentzian in the Fourier domain, with $\sigma>0$ defining the degree of nonlocality. Interestingly, for parametric interaction, the response function can also be periodic, $R(x) \propto$ $\sin (|x| / \sigma)$ in certain regimes of the parameter space [31].

To obtain analytically tractable results the strongly nonlocal limit of $\sigma \rightarrow \infty$ is often used, in which the equation becomes linear [27,32-34] and the solitons are known as accessible solitons [20]. The so-called weakly nonlocal limit $(\sigma \ll 1)$ also presents a simpler model, which can be solved exactly for both dark and bright solitons [35].

Other types of localized response functions have been used to obtain qualitative analytical results that capture the physics of the effect of nonlocality, such as a Gaussian in connection with variational calculations [36,37]. The generic properties of the different types of response functions have been studied by Wyller et al. in terms of modulational instability and it was shown that, in general, all types of localized response functions have the same generic properties, provided their Fourier transform is positive-definite [38].

Here we combine two approaches. First we use the weakly nonlocal model because it allows us to study any localized response function by a single parameter. This allows us to derive the weakly nonlocal form of the interaction potential for any localized response function using the variational approach. Then we introduce an arbitrary degree of nonlocality. We do this by assuming a box-type localized response function because this allows us to calculate the integrals that appear in the variational approach. By comparing the results for arbitrary degree of nonlocality and the box-type response to the generic results obtained in the weakly nonlocal limit we prove that the results are indeed generic.

\section{INTERACTION BETWEEN DARK SOLITONS IN WEAKLY NONLOCAL MEDIUM}

We begin our analysis by considering first the specific weakly nonlocal limit of Eq. (1), in which the width of the response function is much smaller than the spatial scale of the solitons. Then the intensity of the beam $I(\xi, z)$ can be expanded in a Taylor series with respect to $\xi$ around $\xi=x$, and Eq. (1) turns into

$$
i \frac{\partial E}{\partial z}+\frac{1}{2} \frac{\partial^{2} E}{\partial x^{2}}-E\left(I+\gamma \frac{\partial^{2} I}{\partial x^{2}}\right)=0,
$$

where $\gamma=\frac{1}{2} \int_{-\infty}^{+\infty} R(x) x^{2} d x$ clearly shows how the response function needs to be localized. It is important to note that we have here assumed a symmetric response function, which is why it is the second derivative that appears as the perturbation term proportional to $\gamma$. Asymmetric response functions, such as the Raman response in optical fibers, could, of course, easily be used too. However, asymmetric response functions do not allow for defining a Lagrangian and thus to use the variational approach. Thus we consider here only symmetric response functions.
We will investigate the dark solitons using the variational (or Lagrangian) approach [39]. It can be shown that the Lagrangian density corresponding to Eq. (2) is of the following form

$$
\begin{aligned}
\mathcal{L}= & \frac{i}{2}\left(u^{*} \frac{\partial u}{\partial z}-u \frac{\partial u^{*}}{\partial z}\right)\left(1-\frac{1}{|u|^{2}}\right)-\frac{1}{2}\left|\frac{\partial u}{\partial x}\right|^{2} \\
& -\frac{1}{2}\left(|u|^{2}-1\right)^{2}+\frac{1}{2} \gamma\left(\frac{\partial|u|^{2}}{\partial x}\right)^{2},
\end{aligned}
$$

where we normalized the background intensity of the solitons to unity and used the following transformation for the amplitude of the field $E(x, z)=u(x, z) \exp (i z)$. To proceed further we must postulate the form of the function $u(x)$. It was already shown earlier in studies of local dark solitons that the proper ansatz is of the form

$$
u=\left(B \tanh z_{+}-i A\right)\left(B \tanh z_{-}+i A\right),
$$

where $z_{ \pm}=D\left(x \pm x_{0}\right)$ and $2 x_{0}$ denotes the separation between solitons and $A, B$ satisfies the normalization condition $A^{2}+B^{2}=1$. The choice of this particular ansatz is dictated by the fact that it represents exact dark soliton solutions of noninteracting local dark solitons. Substituting Eq. (4) into Eq. (3) and considering the case of weakly overlapping dark solitons, we obtain the averaged Lagrangian $L=\int_{-\infty}^{\infty} \mathcal{L} d x$ in the following form

$$
\begin{aligned}
L= & 2 L_{0}+\frac{d B}{d z} \frac{4 B^{2}}{A D \tanh \left(2 x_{0} D\right)}+16 B^{2} e^{-4 x_{0} D} \\
& \times\left[2 x_{0}\left(D^{2}-B^{2}-4 \gamma B^{2} D^{2}\right)+\frac{B^{2}}{3 D}\left(4 B^{2}-D^{2}\right)\right],
\end{aligned}
$$

where

$$
\begin{aligned}
L_{0}= & 2 \frac{d x_{0}}{d z}\left[-A B+\tan ^{-1}\left(\frac{B}{A}\right)\right] \\
& -\frac{2}{3}\left[B^{2} D+\frac{B^{4}}{D}\right]+\frac{8}{15} \gamma B^{4} D,
\end{aligned}
$$

is the Lagrangian for the noninteracting weakly nonlocal dark solitons [40].

From the corresponding Euler-Lagrangian equations one finds the following relations for soliton parameters

$$
\begin{aligned}
& \frac{d A}{d z}=8 B D e^{-4 x_{0} D}\left[2 x_{0}\left(D^{2}-B^{2}-4 \gamma B^{2} D^{2}\right)\right. \\
& \left.\quad+\frac{B^{2}}{3 D}\left(4 B^{2}-D^{2}\right)\right], \\
& \frac{1}{3}\left[1-\frac{B^{2}}{D^{2}}\right]+16 x_{0} e^{-4 x_{0} D}\left[2 x_{0}\left(D^{2}-B^{2}-4 \gamma B^{2}\right)\right. \\
& \left.+\frac{B^{2}}{3 D}\left(4 B^{2}-D^{2}\right)\right]-\frac{4}{15} \gamma B^{2}=0, \\
& \frac{d x_{0}}{d z}-\frac{A}{3}\left[\frac{D}{B}+\frac{2 B}{D}-\frac{8}{5} \gamma B D\right]+\left[x _ { 0 } \left(D^{2}-2 B^{2}\right.\right. \\
& \left.\left.\quad-8 \gamma B^{2} D^{2}\right)+\frac{B^{2}}{3 D}\left(6 B^{2}-D^{2}\right)\right] \frac{8 A}{B} e^{-4 x_{0} D} \\
& +\frac{d D}{d z}\left[2 D^{2} \tanh \left(2 x_{0} D\right)\right]^{-1}=0 .
\end{aligned}
$$


Assuming well-separated and weakly interacting $\left(x_{0} D \gg 1\right)$ almost "black" solitons $\left(A^{2} \approx 0\right)$ we can obtain from Eqs. (7) through (9) the following equation for the soliton coordinate $x_{0}$

$$
\frac{d^{2} x_{0}}{d z^{2}}=-\frac{d V\left(x_{0}\right)}{d x_{0}},
$$

where we have introduced the "potential" $V\left(x_{0}\right)$ as

$$
V\left(x_{0}\right)=V_{1}\left(x_{0}\right)+V_{2}\left(x_{0}\right),
$$

with

$$
\begin{aligned}
V_{1}\left(x_{0}\right)= & \frac{2\left(1-\frac{16}{15} \gamma B^{2}\right)}{1-\frac{4}{5} \gamma B^{2}} \exp \left(-\frac{4 x_{0} B}{\sqrt{1-\frac{4}{5} \gamma B^{2}}}\right) B^{4}, \\
V_{2}\left(x_{0}\right)= & -\frac{2\left(1-\frac{16}{15} \gamma B^{2}\right)}{1-\frac{4}{5} \gamma B^{2}} \exp \left(-\frac{4 x_{0} B}{\sqrt{1-\frac{4}{5} \gamma B^{2}}}\right) \\
& \times B^{4}\left[\frac{8}{5} \gamma\left(\frac{4 x_{0} B}{\sqrt{1-\frac{4}{5} \gamma B^{2}}}+1+\frac{2}{3} B^{2}\right)\right] .
\end{aligned}
$$

Therefore, for set values of the soliton parameter $B$ and nonlocality $\gamma$, the dynamics of soliton interaction is represented as a mechanical analogy describing the motion of a particle in an external potential. The potential consists of two contributions. The first one, $V_{1}\left(x_{0}\right)$, which exists even for local nonlinearity, is positive and hence is responsible for the naturally occurring dark soliton repulsion $[16,41,42]$. The second contribution, $V_{2}\left(x_{0}\right)$, provides a nonlocality-mediated attractive force, which disappears for $\gamma=0$.

The simultaneous presence of competing repulsive and nonlocality-induced attractive forces introduces a local well in the soliton interaction potential $V\left(x_{0}\right)$, as clearly demonstrated in Fig. 1 for $\gamma=0.05$, which enables the formation of soliton bound states otherwise not possible in the local NLS equation. This result is obtained in the specific weakly nonlocal limit,

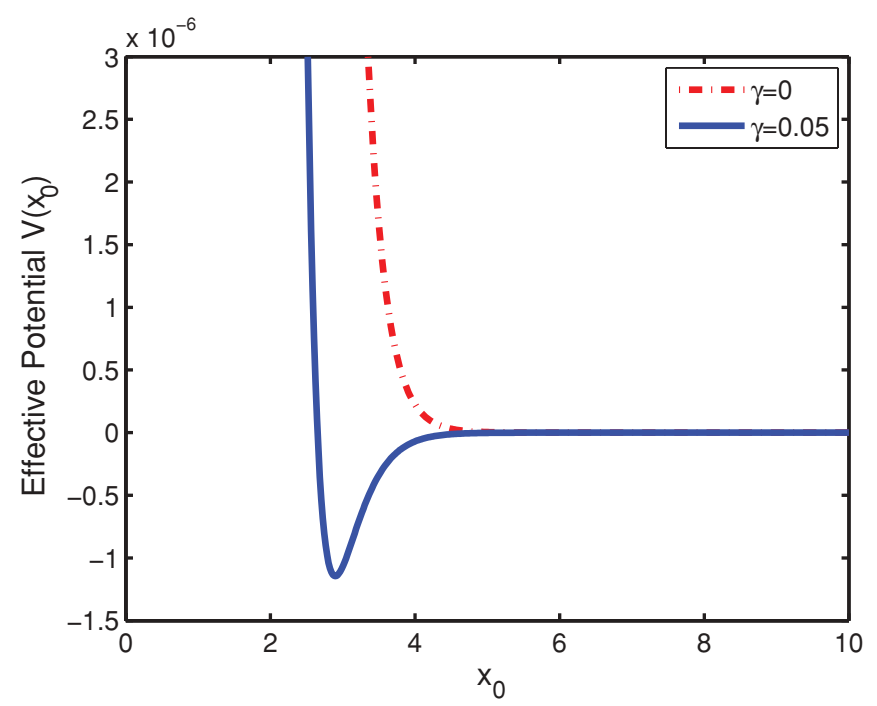

FIG. 1. (Color online) Dark soliton weakly nonlocal interaction potential $V\left(x_{0}\right)$ given by Eq. (11). Red dashed line is the local case $(\gamma=0)$, blue solid line is the weakly nonlocal regime $(\gamma=0.05)$. which has the nice advantage of being generic, in the sense that it is valid for any localized and symmetric response function. In the following section we will extend the results to the full regime of an arbitrary degree of nonlocality by considering a specific response function. However, we connect the general results to the generic result of the weakly nonlocal limit to demonstrate the generic nature of also the general result.

\section{GENERAL NONLOCAL CASE}

Here we consider the interaction between the dark solitons in nonlocal media, in which the nonlocal response has an arbitrary degree of nonlocality. Then the Lagrangian density corresponding to Eq. (1) is

$$
\begin{aligned}
\mathcal{L}= & \frac{i}{2}\left(u^{*} \frac{\partial u}{\partial z}-u \frac{\partial u^{*}}{\partial z}\right)\left(1-\frac{1}{|u|^{2}}\right)-\frac{1}{2}\left|\frac{\partial u}{\partial x}\right|^{2} \\
& -\frac{1}{2}\left(|u|^{2}-1\right) \int_{-\infty}^{+\infty} R(x-\xi)\left[|u(\xi, z)|^{2}-1\right] d \xi
\end{aligned}
$$

To make the problem analytically tractable we will consider here the particular model of nonlocality described by the rectangular nonlocal response function

$$
R(x)= \begin{cases}\frac{1}{2 \sigma} & -\sigma \leqslant x \leqslant \sigma \\ 0 & \text { otherwise }\end{cases}
$$

Physically, this type of nonlocal response means that the nonlinear response of the medium in a particular spatial location is determined by the equal contributions from the light intensity in the neighborhood of this location defined by parameter $\sigma$. This is obviously a simplification, but as we will see later, it leads to a physically correct description of the soliton interaction.

Substituting Eq. (4) into Eq. (12) and integrating over the transverse coordinate $x$, we obtain the averaged Lagrangian in the form

$$
\begin{aligned}
L= & \frac{d B}{d z} \frac{4 B^{2}}{A D \tanh \left(2 x_{0} D\right)}+4 \frac{d x_{0}}{d z}\left[-A B+\tan ^{-1}\left(\frac{B}{A}\right)\right] \\
& -\frac{4}{3} B^{2} D\left[4 B^{2}+12-24 D x_{0}\right] e^{-4 x_{0} D}-\frac{4}{3} B^{2} D \\
& +\frac{2 B^{4}}{D}\left[\operatorname{csch}^{2}(D \sigma)-\frac{\operatorname{coth}(D \sigma)}{D \sigma}\right]+\frac{4 B^{4}}{D}[2 \cosh (2 D \sigma) \\
& -\left(4 D x_{0}-1\right) \frac{\sinh (2 D \sigma)}{D \sigma}+8 B^{2}+4 B^{2} \operatorname{csch}^{2}(D \sigma) \\
& \left.-\frac{4 B^{2} \operatorname{coth}(D \sigma)}{D \sigma}\right] e^{-4 x_{0} D} .
\end{aligned}
$$

From the corresponding Euler-Lagrangian equations one can derive the evolution equation for the soliton coordinate, which in the limit of weakly interacting (i.e., well separated), 
almost black solitons $\left(A^{2} \ll 1\right)$ takes the following form

$$
\begin{aligned}
\frac{d^{2} x_{0}}{d z^{2}}= & {\left[\frac{D}{3 B}-\frac{B}{D}\left(\operatorname{csch}^{2}(D \sigma)-\frac{\operatorname{coth}(D \sigma)}{D \sigma}\right)\right] } \\
& \times\left\{\frac{2}{3} B D^{2}\left(24 D x_{0}-4 B^{2}-12\right)\right. \\
& +2 B^{3}\left[\left(1-4 D x_{0}\right) \frac{\sinh (2 D \sigma)}{D \sigma}+2 \cosh (2 D \sigma)\right. \\
& \left.\left.+8 B^{2}+4 B^{2} \operatorname{csch}^{2}(D \sigma)-\frac{4 B^{2} \operatorname{coth}(D \sigma)}{D \sigma}\right]\right\} e^{-4 x_{0} D} \\
= & -\frac{d V\left(x_{0}\right)}{d x_{0}},
\end{aligned}
$$

where the effective potential function $V\left(x_{0}\right)$ is defined as

$$
\begin{aligned}
V\left(x_{0}\right)= & {\left[\frac{D^{2}}{3}-B^{2}\left(\operatorname{csch}^{2}(D \sigma)-\frac{\operatorname{coth}(D \sigma)}{D \sigma}\right)\right] } \\
& \times\left\{\left(1+4 D x_{0}\right)\left(1-\frac{B^{2}}{2 D^{2}} \frac{\sinh (2 D \sigma)}{D \sigma}\right)-\frac{2}{3}\left(B^{2}+3\right)\right. \\
& +\frac{B^{2}}{2 D^{2}}\left[\frac{\sinh (2 D \sigma)}{D \sigma}+2 \cosh (2 D \sigma)+8 B^{2}\right. \\
& \left.\left.+4 B^{2} \operatorname{csch}^{2}(D \sigma)-\frac{4 B^{2} \operatorname{coth}(D \sigma)}{D \sigma}\right]\right\} e^{-4 x_{0} D},
\end{aligned}
$$

and parameters $B, D$, and $\sigma$ satisfy the following relation

$$
\frac{1}{3}-\frac{B^{2}}{D^{2}} \frac{\operatorname{coth}(D \sigma)}{D \sigma}\left[1-D^{2} \sigma^{2} \operatorname{csch}^{2}(D \sigma)\right]=0 .
$$

One can show that in the weakly nonlocal limit (i.e., when $\sigma \ll 1)$ the formula Eq. (16) leads to the potential of the form of Eq. (11) with the nonlocality parameter $\gamma$ given by $\gamma=\frac{1}{2} \int_{-\infty}^{+\infty} R(x) x^{2} d x=\sigma^{2} / 6$. In Fig. 2 we show the potential $V\left(x_{0}\right)$ for different values of the nonlocality $\sigma$. It is

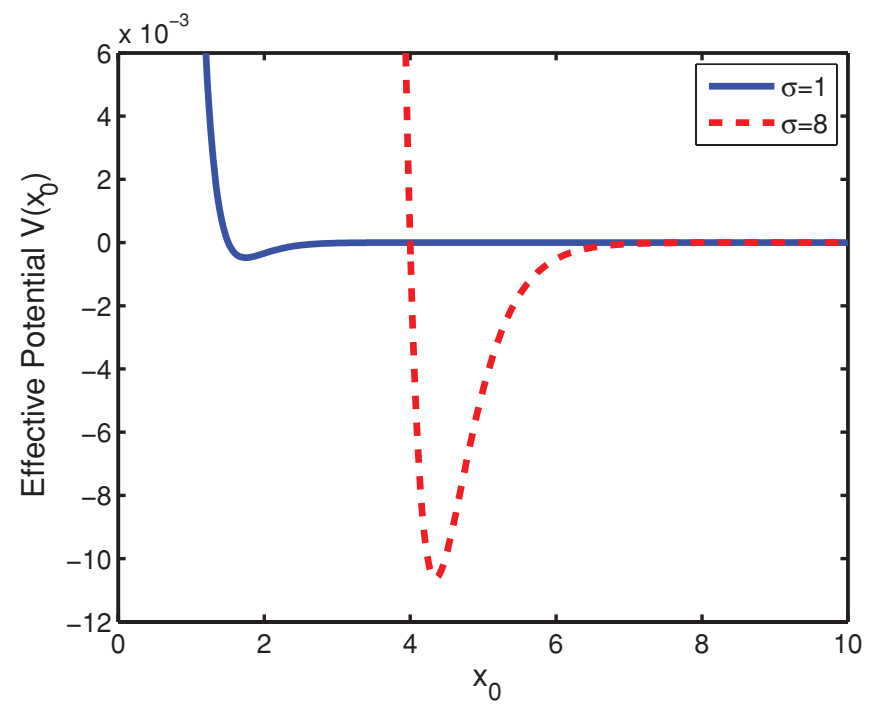

FIG. 2. (Color online) Dark soliton interaction potential $V\left(x_{0}\right)$, given by Eq. (16), for a rectangular nonlocal response with an arbitrary degree of nonlocality $\sigma$. Blue solid line is $(\sigma=1)$; red dashed line is the strongly nonlocal regime $(\sigma=8.0)$.
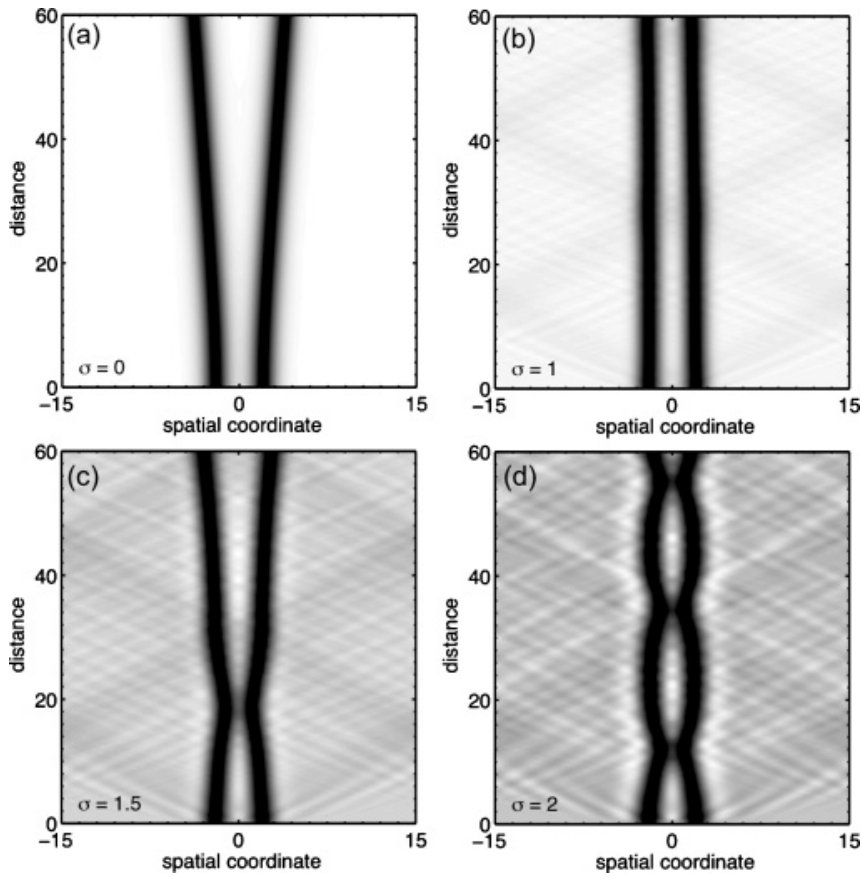

FIG. 3. Numerically simulated interaction of initially parallel dark solitons in nonlocal medium with rectangular nonlocal response function, for different degree of nonlocality (a) $\sigma=0$, (b) $\sigma=1$, (c) $\sigma=1.5$, and (d) $\sigma=2.0$. Notice the almost parallel propagation of solitons for $\sigma=1$, indicating the balance between repulsive and attractive forces and consequently formation of the soliton bound state.

evident that the generic results of the weakly nonlocal model remain valid also for an arbitrary degree of nonlocality, that is, nonlocality provides an attractive contribution to the potential, which counteracts the natural repulsion of dark solitons thus enabling the formation of their bound states. This fact provides evidence that our general results for the specific rectangular response function are, in fact, generic also for any symmetric and localized response function.

We now confirm our theory by direct numerical simulations of the nonlocal NLS Eq. (1) with the rectangular nonlocal response function. As initial conditions we used Kerr soliton profiles [see Eq. (4)] with $A=0, B=D=1$. The representative results are depicted in Fig. 3. These contour plots show the dynamics of initially well-separated solitons. The separation is chosen to be sufficiently small so that both solitons clearly repel when the nonlinearity is local [Fig. 3(a)]. It is clear that as the extent of nonlocal response increases both solitons start experiencing the attractive force. In fact, in the case depicted in Fig. 3(b) $(\sigma=1)$ the natural repulsion of solitons is almost completely compensated for by the nonlocality-mediated attraction leading to the formation of the bound state of dark solitons. Interestingly, in this case the solitons are separated by the distance of $2 x_{0}=3.8$, which corresponds to the location of the minimum of the effective potential from Fig. 2 for $\sigma=1$. For even stronger nonlocality the attractive force causes mutual oscillations of the solitons' trajectories. The radiation visible in Figs. 3(b) through 3(d) is a result of the fact that the initial wave profiles are not exact 


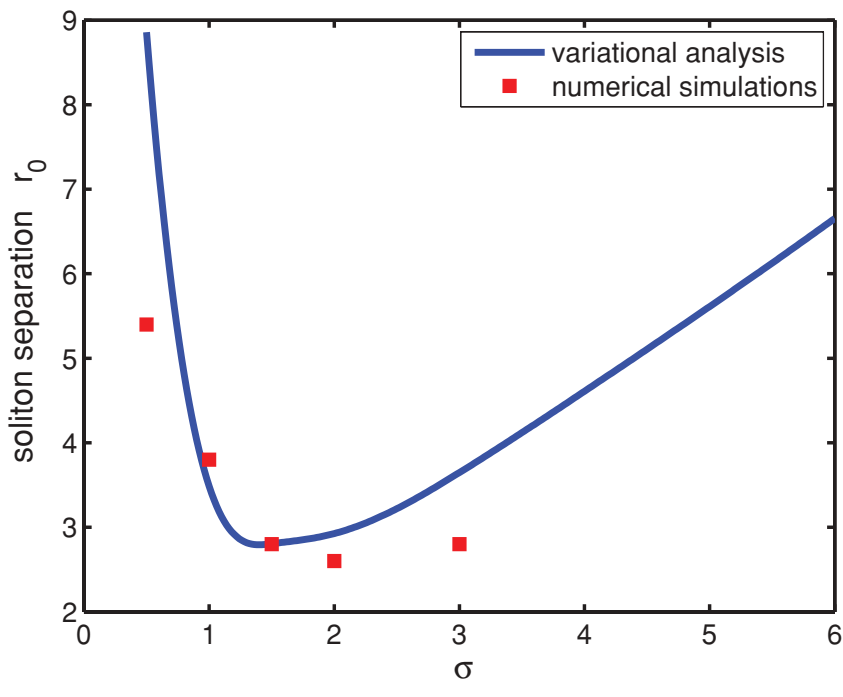

FIG. 4. (Color online) Separation between solitons in a bound state $\left(r_{0}\right)$ as a function of the degree of nonlocality $\sigma$. Solid line represents the variational calculations; squares are the numerical simulations of soliton propagation.

dark solitons in the nonlocal regime. Hence the solitons evolve and transform as they propagate shading away radiation.

In Fig. 4 we plot with the solid line the separation between solitons corresponding to their bound state as calculated from the minimum of the effective potential Eq. (17). It is evident that the separation is a nonmonotonic function of the degree of nonlocality. This can be explained as follows. For the local case $(\sigma=0)$ the separation must tend to infinity because of the sole presence of the local repulsive potential. As $\sigma$ increases from zero the nonlocal attractive force comes to play and thus the soliton separation will decrease until the balance between repulsion and attraction is reached. The soliton separation continues to decrease with increasing $\sigma$ until it becomes comparable with the degree of nonlocality. For larger $\sigma$ the resulting nonlinearity-induced waveguide becomes wider and shallower. Since the soliton bound state must still represent the mode of this waveguide it must increase its spatial extent leading to bigger separation. This behavior has been confirmed in numerical simulations. To this end, for a given degree of nonlocality, we varied the initial distance between the solitons and numerically propagated them over a distance long enough to establish the formation of their bound state. The resulting separation is depicted in Fig. 4 by filled squares. Clearly it follows the trend found from variational analysis. On the other hand, the numerical data are limited to a relatively low degree of nonlocality because the strong radiation for larger $\sigma$ prevents the accurate determination of the bound states.

\section{CONCLUSION}

We studied analytically the interaction of dark spatial solitons in a nonlocal medium. We used a variational technique to derive the evolution equations for the separation between the solitons. We showed that nonlocality provides an attractive force between otherwise repelling solitons and enables the formation of their bound states. The resulting soliton separation is determined by the degree of nonlocality, but cannot be smaller than the width of the nonlocal response. Our theoretical results have been confirmed by direct numerical simulations of the nonlinear nonlocal Schrödinger equation.

\section{ACKNOWLEDGMENTS}

This work was supported by the National Natural Science Foundation of China (Grant No. 60808002), the Shanghai Leading Academic Discipline Program (Grant No. S30105), the China Scholarship Council, and the Australian Research Council.
[1] Yu. S. Kivshar and G. P. Agrawal, Optical Solitons: From Fibers to Photonic Crystals (Academic, New York, 2003).

[2] Y. S. Kivshar and B. Luther-Davies, Phys. Rep. 298, 81 (1998) and references therein.

[3] S. R. Skinner, G. R. Allan, D. R. Andersen, and A. L. Smirl, IEEE J. Quantum Electron. 27, 2211 (1991).

[4] G. A. Swartzlander, Jr., D. R. Andersen, J. J. Regan, H. Yin, and A. E. Kaplan, Phys. Rev. Lett. 66, 1583 (1991).

[5] A. Hasegawa and F. Tappert, Appl. Phys. Lett. 23, 171 (1973).

[6] W. J. Tomlinson, R. J. Hawkins, A. M. Weiner, J. P. Heritage, and R. N. Thurston, J. Opt. Soc. Am. B 6, 329 (1989).

[7] S. Burger, K. Bongs, S. Dettmer, W. Ertmer, K. Sengstock, A. Sanpera, G. V. Shlyapnikov, and M. Lewenstein, Phys. Rev. Lett. 83, 5198 (1999).

[8] R. Nath, P. Pedri, and L. Santos, Phys. Rev. Lett. 101, 210402 (2008).

[9] S. Stellmer, C. Becker, P. Soltan-Panahi, E.-M. Richter, S. Dörscher, M. Baumert, J. Kronjäger, K. Bongs, and K. Sengstock, Phys. Rev. Lett. 101, 120406 (2008).
[10] A. Weller, J. P. Ronzheimer, C. Gross, J. Esteve, M. K. Oberthaler, D. J. Frantzeskakis, G. Theocharis, and P. G. Kevrekidis, Phys. Rev. Lett. 101, 130401 (2008).

[11] C. Milián, D. V. Skryabin, and A. Ferrando, Opt. Lett. 34, 2096 (2009).

[12] J. P. Gordon, Opt. Lett. 8, 596 (1983).

[13] M. Shalaby and A. Barthelemy, Opt. Lett. 16, 1472 (1991).

[14] J. S. Aitchison, A. M. Weiner, Y. Silberberg, D. E. Leaird, M. K. Oliver, J. L. Jackel, and P. W. E. Smith, Opt. Lett. 16, 15 (1991).

[15] K. J. Blow and N. J. Doran, Phys. Lett. A 107, 55 (1985).

[16] W. Zhao and E. Bourkoff, Opt. Lett. 14, 1371 (1989).

[17] D. Foursa and P. Emplit, Phys. Rev. Lett. 77, 4011 (1996).

[18] N. I. Nikolov, D. Neshev, W. Krolikowski, O. Bang, J. J. Rasmussen, and P. L. Christiansen, Opt. Lett. 29, 286 (2004).

[19] A. Dreischuh, D. N. Neshev, D. E. Petersen, O. Bang, and W. Krolikowski, Phys. Rev. Lett. 96, 043901 (2006).

[20] A. W. Snyder and D. J. Mitchell, Science 276, 1538 (1997). 
[21] S. Skupin, O. Bang, D. Edmundson, and W. Krolikowski, Phys. Rev. E 73, 066603 (2006).

[22] O. Bang, W. Krolikowski, J. Wyller, and J. J. Rasmussen, Phys. Rev. E 66, 046619 (2002).

[23] M. Peccianti, K. A. Brzdakiewicz, and G. Assanto, Opt. Lett. 27, 1460 (2002).

[24] P. D. Rasmussen, O. Bang, and W. Krolikowski, Phys. Rev. E 72, 066611 (2005).

[25] W. Hu, T. Zhang, Q. Guo, L. Xuan, and S. Lan, Appl. Phys. Lett. 89, 071111 (2006).

[26] N. Ghofraniha, C. Conti, G. Ruocco, and S. Trillo, Phys. Rev. Lett. 99, 043903 (2007).

[27] N. I. Nikolov, D. Neshev, O. Bang, and W. Z. Krolikowski, Phys. Rev. E 68, 036614 (2003).

[28] P. V. Larsen, M. P. Sørensen, O. Bang, W. Z. Krolikowski, and S. Trillo, Phys. Rev. E 73, 036614 (2006).

[29] M. Bache, O. Bang, J. Moses, and F. W. Wise, Opt. Lett. 32, 2490 (2007).

[30] M. Bache, O. Bang, W. Krolikowski, J. Moses, and F. W. Wise, Opt. Express 16, 3273 (2008).

[31] W. Krolikowski, O. Bang, N. I. Nikolov, D. Neshev, J. Wyller, J. J. Rasmussen, and D. Edmundson, J. Opt. B 6, S288 (2004).

[32] C. Conti, M. Peccianti, and G. Assanto, Phys. Rev. Lett. 92, 113902 (2004).
[33] D. Deng, Q. Guo, and W. Hu, Phys. Rev. A 79, 023803 (2009); W.-P. Zhong and M. Belić, ibid. 79, 023804 (2009); D. Deng and Q. Guo, J. Opt. A: Pure Appl. Opt. 10, 035101 (2008).

[34] I. V. Shadrivov and A. A. Zharov, J. Opt. Soc. Am. B 19, 596 (2002).

[35] W. Krolikowski and O. Bang, Phys. Rev. E 63, 016610 (2000).

[36] M. Shen, N. Xi, Q. Kong, L.-J. Ge, J.-L. Shi, and Q. Wang, Chin. Phys. B 18, 2822 (2009).

[37] D. Briedis, D. E. Petersen, D. Edmundson, W. Krolikowski, and O. Bang, Opt. Express 13, 435 (2005); A. I. Yakimenko, V. M. Lashkin, and O. O. Prikhodko, Phys. Rev. E 73, 066605 (2006); S. Skupin, M. Grech, and W. Krolikowski, Opt. Express 16, 9118 (2008).

[38] J. Wyller, W. Krolikowski, O. Bang, and J. J. Rasmussen, Phys. Rev. E 66, 066615 (2002).

[39] D. Anderson, Phys. Rev. A 27, 3135 (1983).

[40] L. J. Ge, Q. Wang, M. Shen, J. L. Shi, Q. Kong, and P. Hou, J. Opt. A: Pure Appl. Opt. 11, 065207 (2009).

[41] Y. S. Kivshar and W. Krolikowski, Opt. Commun. 114, 353 (1995).

[42] G. Theocharis, P. Schmelcher, M. K. Oberthaler, P. G. Kevrekidis, and D. J. Frantzeskakis, Phys. Rev. A 72, 023609 (2005). 